Ann. Biol. anim. Bioch. Biophys., I976, 16 (5), 719-729.

\title{
INHIBITION DE LA PROTEOLYSE PEPSIQUE IN VITRO PAR LE BLÉ. RÔLE DE L'ACIDE PHYTIQUE DES ISSUES
}

\author{
Marie-Claire CAMUS et J. C. LAPORTE \\ avec la collaboration technique de P. Pocholle \\ Unité de Recherches diététiques de l'I.N.S.E.R.M., \\ Hôpital Bichat, \\ 170, Boulevard Ney, \\ 75018 Paris
}

RÉSUMÉ

Nous avons étudié la protéolyse pepsique in vitro de caséine ou de gluten, seuls ou additionnés soit de blé Étoile de Choisy, soit d'extraits aqueux de ce même blé ou de ses différentes fractions de meunerie. La protéolyse est suivie à $\mathrm{pH} 2,0$ par la mesure à $280 \mathrm{~nm}$ de la densité optique des produits solubles dans une solution éthanolique o, I M en acide acétique et acétate de sodium. Le blé et ses extraits acides entraînent une inhibition de la protéolyse tant de la caséine que du gluten, de même que les extraits acides de gros son, de son fin mais non de farine à $65 \mathrm{p}$. Ioo. Cette inhibition augmente avec la concentration d'inhibiteur et diminue lorsque celle de substrat augmente, mais est indépendante de celle de pepsine. Les blés Rex et $28 p C$ rouge donnent lieu au même phénomène. L'acide phytique pur produit un effet analogue. Or, dans le blé, il est surtout présent dans les issues. Il paraît donc être l'agent de l'inhibition de la protéolyse produite par le blé. Son action s'explique par la formation au pH acide où s'effectue la protéolyse, de composés insolubles de phytates de protéines, plus résistants que les protéines à l'action de la pepsine.

\section{INTRODUCTION}

Des expériences de protéolyses comparées in vitro de divers blés et de gluten, avaient montré que celui-ci donnait lieu à des valeurs plus importantes (CAmus et SAUTIER, I972). En vue d'expliquer ce phénomène, nous avons recherché une inhibition éventuelle de la protéolyse pepsique tant du gluten que de la caséine en présence de blé ou de certaines de ses fractions de meunerie. Nous avons d'abord étudié l'influence du produit de mouture intégrale puis de ses extraits aqueux effectués à divers $\mathrm{pH}$, enfin des extraits aqueux des différentes fractions. Ayant de fait observé 
une inhibition de la protéolyse pepsique dans certains cas, nous avons été amenés à imputer cet effet à l'acide phytique présent principalement dans les issues (GuILIEMET, I946; KENT-JONES et AMOS, I950) et nous avons étudié l'influence de l'acide phytique pur sur cette protéolyse.

\section{MATÉRIEI ET MÉTHODES}

La protéolyse pepsique de la caséine et du gluten est suivie en mesurant à $280 \mathrm{~nm}$ la densité optique des produits solubles dans l'éthanol acidifié (LAPORTE, 1964).

\section{Mode opératoire}

La protéolyse est faite en tubes à essai $\mathrm{I}_{4} \times \mathrm{I}_{4} \mathrm{O} \mathrm{mm}$ sous agitation magnétique à $37^{\circ} \mathrm{C}$, $\mathrm{pH} 2,0$, pendant 60 minutes. Le volume du milieu réactionnel est de $1 \mathrm{ml}$ comprenant :

\section{Substrat.}

- Soit o, I à $0,8 \mathrm{ml}$ d'une solution amenée à $\mathrm{pH}$ 2,0 par $\mathrm{HCl}$, de caséine Merck (d'après HAMMARSTEN) à $50 \mathrm{mg} / \mathrm{ml}$.

- Soit ro à $40 \mathrm{mg}$ de gluten de blé Étoile de Choisy et 0,2 à $0,8 \mathrm{ml}$ d'une solution d' $\mathrm{HCl}$ à une concentration telle que le $\mathrm{pH}$ soit de 2,0 .

\section{Effecteur.}

- Soit o à $200 \mathrm{mg}$ du blé étudié (Étoile de Choisy ou Rex ou $28 p C$ rouge, ce dernier étant constitué d'un mélange de Capelle, Capitole, Rémois, Hardy), utilisé après broyage au moulin à café électrique des grains entiers.

- Soit o à $0,7 \mathrm{ml}$ d'un extrait préparé comme suit : $15 \mathrm{~g}$ de blé broyé comme précédemment ou les quantités correspondantes des différentes fractions de meunerie $(3,90 \mathrm{~g}$ de gros son, $\mathrm{I}, 35 \mathrm{~g}$ de son fin, 9,75 $\mathrm{g}$ de farine au taux d'extraction de $65 \mathrm{p}$. Ioo) sont mis en suspension à $o^{\circ} \mathrm{C}$, sous agitation magnétique pendant Io minutes dans $30 \mathrm{ml}$ d'une solution d' $\mathrm{HCl}$ à une concentration telle que le $\mathrm{pH}$ final soit celui désiré. Puis la suspension est homogénéisée pendant $\mathrm{I}$ minute à l'Ultra-Turrax et centrifugée pendant to minutes à $15000 \mathrm{~g}$. Le surnageant constitue l'extrait utilisé.

- Soit $0,5 \mathrm{ml}$ d'une solution amenée à $\mathrm{pH}$ 2,0 par $\mathrm{HCl}$, de phytate de sodium Sigma (contenant $12 \mathrm{Na}$ par mole et $4 \mathrm{I}, 3 \mathrm{p}$. IOO d' $\mathrm{H}_{2} \mathrm{O}$ ) à une concentration de $\mathrm{I}$ à $20 \mathrm{mg} / \mathrm{ml}$.

\section{Enzyme.}

o, I ml d'une solution dans $\mathrm{HCl}$ o, or $\mathrm{N}$ de pepsine cristallisée Worthingthon à $3000 \mathrm{U} / \mathrm{mg}$, à une concentration de ro à $40 \mu \mathrm{g} / \mathrm{ml}$.

Le substrat et l'effecteur sont préincubés pendant to minutes à $37^{\circ} \mathrm{C}$, le mélange ayant été amené à un volume de $0,9 \mathrm{ml}$ et à un $\mathrm{pH}$ de 2,0 par une solution convenable d' $\mathrm{HCl}$. Au temps $\mathrm{o}$, on ajoute l'enzyme. Au temps 60 minutes, la réaction est arrêtée par adjonction de $9 \mathrm{ml}$ d'unc solution éthanolique o, $\mathbf{~ M}$ en acide acétique et acétate de sodium préparée à partir d'éthanol à $95^{\circ} \mathrm{GL}$. Après Io à 15 minutes à la température ambiante, on filtre, mesure la densité optique par $\mathrm{cm}$ du filtrat à $280 \mathrm{~nm}$ et on en retranche celle correspondant au temps $\mathrm{o}$.

\section{RÉSULTATS}

\section{I. - Protéolyse de la caséine et du gluten seuls (fig. I)}

Une inhibition par excès de substrat se manifeste pour la caséine mais non pour le gluten. 

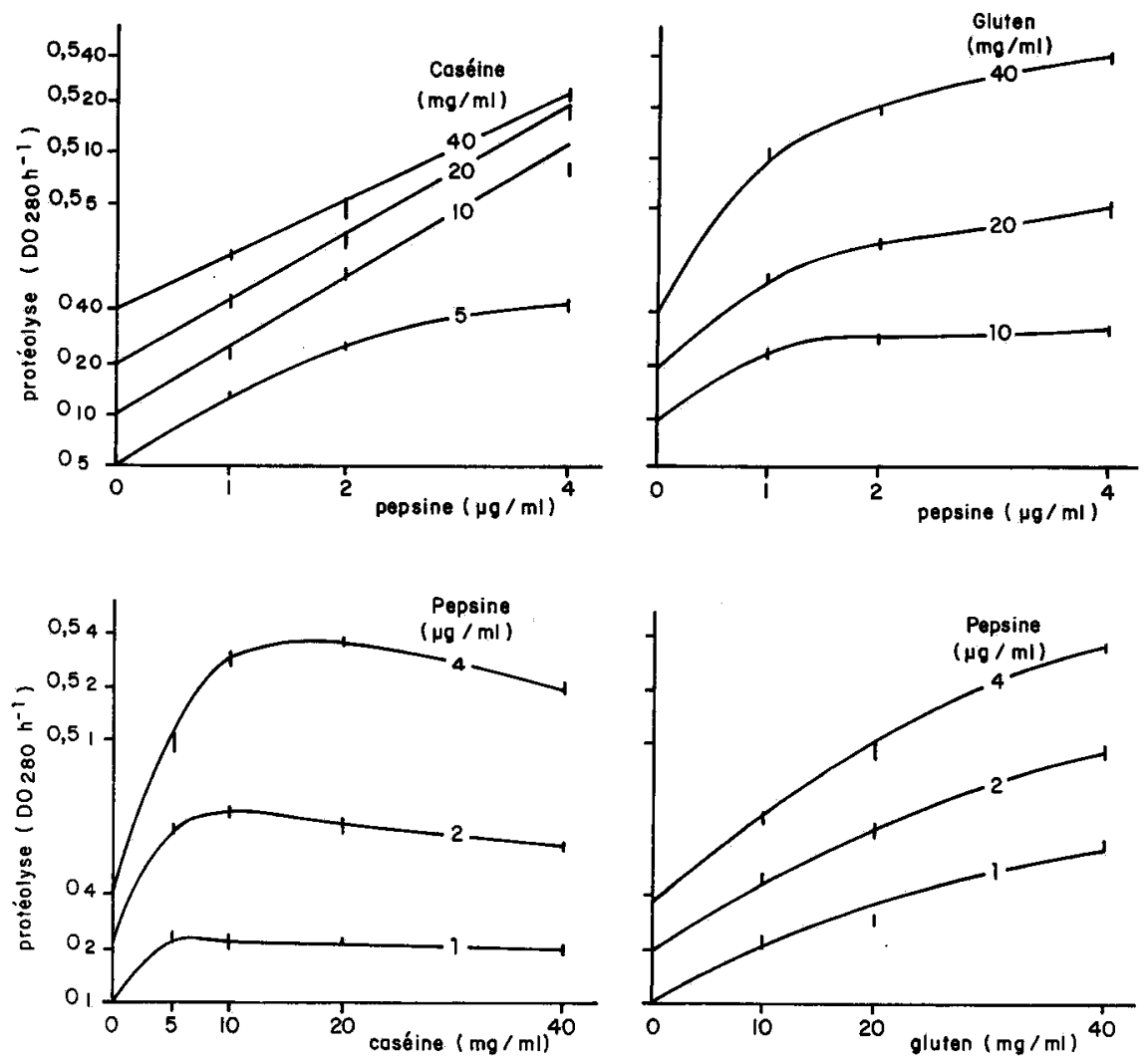

Fig. I. - Protéolyse par la pepsine de caséine ou de gluten seuls

$$
m \pm s_{m}(n=6)
$$

Haut : A concentration constante de substrat

Bas : A concentration constante d'enzyme

\section{2. - Protéolyse d'un mélange de caséine}

ou de gluten et de blé Étoile de Choisy (fig. 2)

Lorsque la concentration de blé augmente, la protéolyse d'abord diminue, passe par un minimum, puis augmente, cette augmentation étant plus précoce et plus importante dans le cas du gluten que de la caséine où elle est très peu marquée.

\section{3. - Protéolyse d'un mélange de caséine}

ou de gluten et d'extraits aqueux de blé Étoile de Choisy

a) Influence du pH d'extraction (fig. 3).

En présence des extraits effectués aux $\mathrm{pH}$ les plus bas, la protéolyse du mélange est très inférieure à celle de la caséine ou du gluten seuls. Lorsque le $\mathrm{pH}$ d'extraction augmente :

- dans le cas de la caséine, la protéolyse du mélange reste faible jusqu'à pH 3,0, puis augmente pour atteindre à $\mathrm{pH} 7,0$ la valeur obtenue avec la seule caséine ; 
Fig. 2

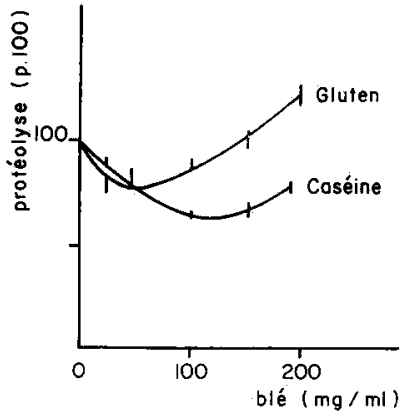

Fig. 3

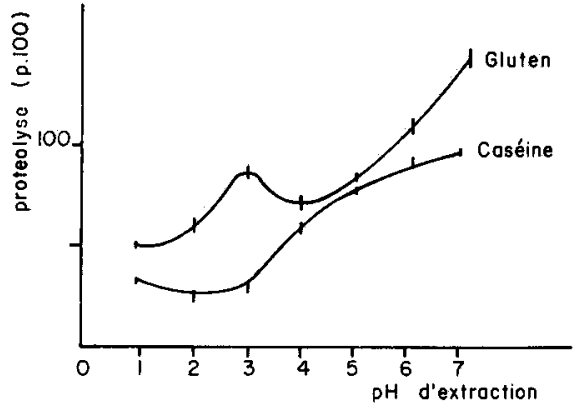

FIG. 2. - Protélyse par la pepsine $(4 \mu \mathrm{g} / \mathrm{ml})$ d'un mélange de caséine ou de gluten (ro $\mathrm{mg} / \mathrm{ml}$ ) et de blé Étoile de Choisy (en p. Ioo de la protéolyse de la caséine ou du gluten seuls).

$$
m \pm s_{m}(n=6)
$$

FIG. 3. - Protéolyse par la pepsine $(4 \mu \mathrm{g} / \mathrm{ml})$ d'un mélange de caséine ou de gluten (Io $\mathrm{mg} / \mathrm{ml}$ ) et d'extraits aqueux effectués à divers $p H$, de blé Étoile de Choisy $(200 \mathrm{mg} / \mathrm{ml}$ ) (en $\mathrm{p}$. roo de la protéolyse de la caséine ou du gluten seuls).

$$
m \pm s_{m}(n=6)
$$

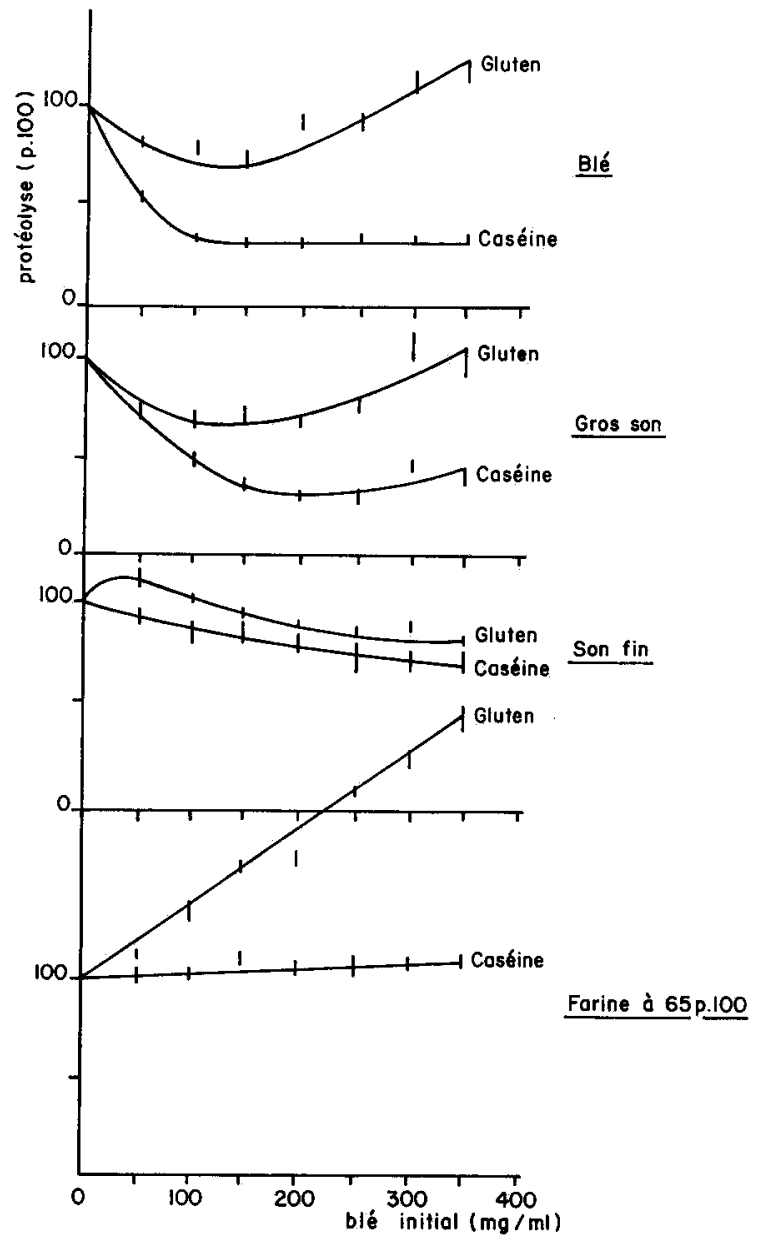

I'ı́. 4. - Protéolyse par la pepsine (4 $\mu \mathrm{g} / \mathrm{ml})$ d'un mélange de caséiné ou de gluten (Io $\mathrm{mg} / \mathrm{ml}$ ) et d'extraits aqueux effectués à $p H$ 2,0 de différentes fractions de blé (en p. Ioo de la protéolyse de la caséine ou du gluten seuls).

$$
m \pm s_{m}(n=6)
$$


- dans le cas du gluten, la protéolyse du mélange augmente pour atteindre à $\mathrm{pH} 7,0$ des valeurs supérieures à celles obtenues avec le seul gluten. Cette augmentation n'est pas régulière dans tout l'intervalle de $\mathrm{pH}$ étudié, mais présente un maximum intermédiaire à $\mathrm{pH}_{3,0}$.

b) Infuence des extraits à $p H$ 2,0 de différentes fractions (fig. 4).

A concentration constante d'enzyme et de substrat, en présence d'extraits effectués à $\mathrm{pH} 2, \mathrm{o}$ de blé ou de ses différentes fractions de meunerie, à concentration variable, 1'accroissement de la concentration de l'extrait de blé provoque une diminution de la protéolyse, conduisant à un plateau pour la caséine, et pour le gluten à une remontée après passage par un minimum. Cet effet se retrouve presque identique avec l'extrait de gros son correspondant. Avec l'extrait de son fin, on n'observe dans les deux cas qu'une seule phase de diminution, plus faible et régulière. Par contre, alors que l'extrait de farine à $65 \mathrm{p}$. roo est sans effet dans le cas de la caséine, il entraîne une augmentation de la protéolyse dans le cas du gluten.

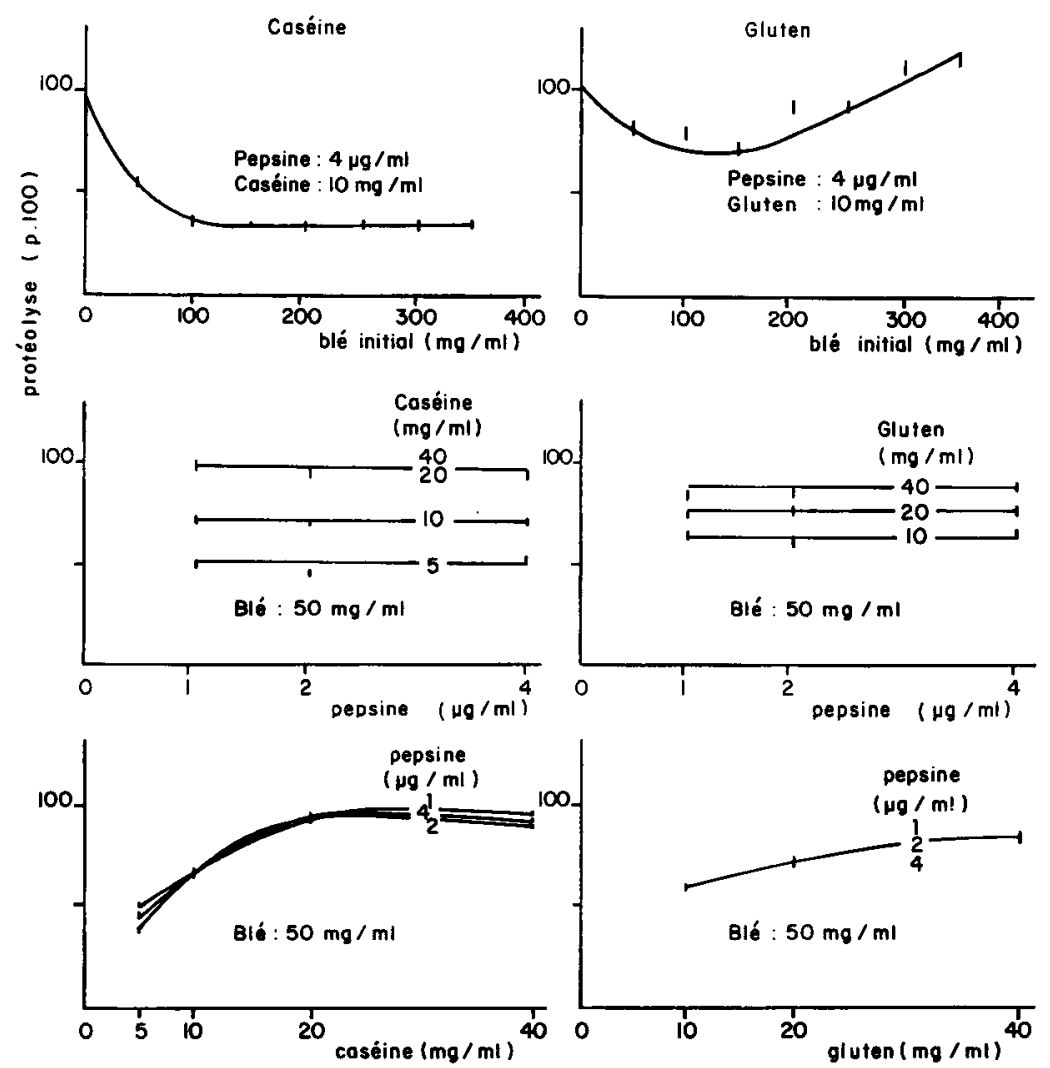

Fıc. 5. - Proteolyse par la pepsine d'un mélange de caséine ou de gluten et d'un extrait aqueux effectué à pH 2,0 de blé Étoile de Choisy (en p. Ioo de la protéolyse de la caséine ou du gluten seuls).

$$
m \pm s_{m}(n=6)
$$


c) Étude systématique de l'effet obtenu avec l'extrait à pH 2,0 du blé (fig. 5).

- A concentration constante d'enzyme et de substrat, l'augmentation de la concentration d'extrait entraîne une diminution de la protéolyse, conduisant à un plateau pour la caséine et à une remontée après passage par un minimum pour le gluten (cf. 3, b).

- A concentration constante de substrat et d'extrait, le rapport des protéolyses en présence et en absence d'extrait, est inférieur à $I$ et indépendant de la concentration d'enzyme.

- A concentration constante d'enzyme et d'extrait, ce même rapport, tout en restant inférieur à $\mathbf{I}$, augmente avec la concentration de substrat.
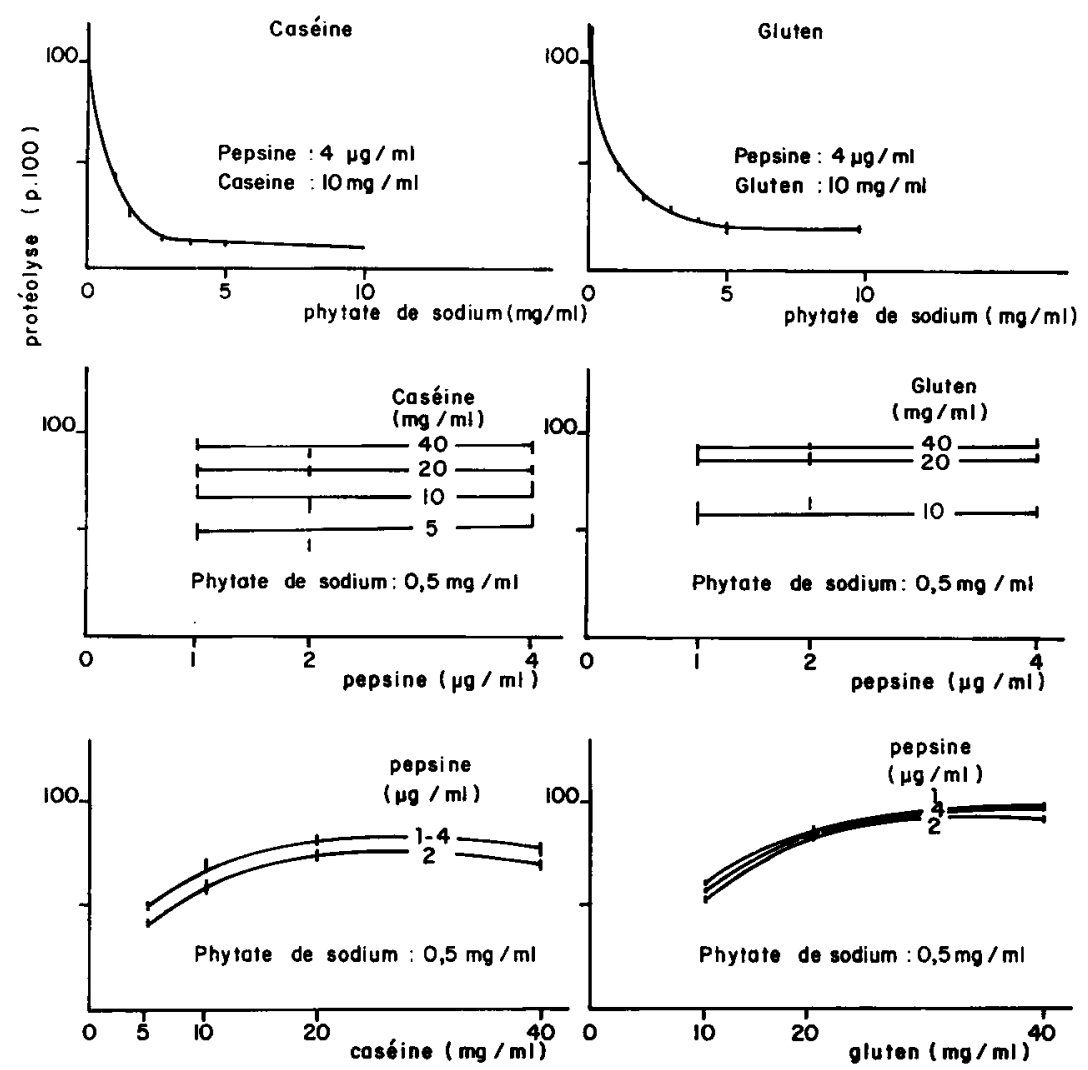

FIG. 6. - Protélyse par la pepsine d'un mélange de caséine ou de gluten et d'acide phytique (en p. Ioo de la protéolyse de la caséine ou du gluten seuls).

$$
m \pm s_{m}(n=6)
$$

\section{4. - Protéolyse d'un mélange de caséine ou de gluten et d'acide phytique (fig. 6)}

- A concentration constante d'enzyme et de substrat, l'augmentation de la concentration d'acide phytique entraîne une diminution de la protéolyse conduisant à un plateau. 
- A concentration constante de substrat et d'acide phytique, le rapport des protéolyses en présence et en absence d'acide phytique, est inférieur à $I$ et indépendant de la concentration d'enzyme.

- A concentration constante d'enzyme et d'acide phytique, ce même rapport, tout en restant inférieur à $I$, augmente avec la concentration de substrat.

\section{5. - Extension de l'étude à d'autres blés}

Pour tester la généralité des résultats obtenus avec le blé Étoile de Choisy, nous avons repris certaines des expériences précédentes avec deux autres blés (Rex et $28 p C$ rouge), limitant toutefois cette étude à la seule caséine. Les résultats obtenus pour la protéolyse d'un mélange de caséine et de chacun de ces blés ou des extraits aqueux de leurs différentes fractions, sont analogues à ceux obtenus avec le blé Étoile de Choisy.

\section{DISCUSSION}

En ce qui concerne la méthode ici utilisée pour l'étude de la protéolyse, elle est basée sur l'insolubilité des protéines non dégradées et la solubilité de leurs produits de dégradation en milieu éthanolique. Or, il est bien connu qu'une fraction des protéines de blé, la gliadine, y est soluble d'une manière d'ailleurs variable selon le degré éthanolique avec un maximum situé vers $70-80^{\circ} \mathrm{GL}$ (BOURDET, I956). Dans nos conditions, la concentration éthanolique est d'environ $85^{\circ} \mathrm{GL}$, donc plus élevée. De plus, il est connu que 1'acide acétique solubilise plus ou moins les protéines du gluten (BouRder, 1956). Quoi qu'il en soit, nous avons constaté que, dans nos conditions de précipitation, $20 \mathrm{p}$. Ioo des protéines du gluten étaient solubilisées et $2 \mathrm{p}$. roo dans le cas de la caséine. Les résultats obtenus pour le gluten ne concernent donc que $80 \mathrm{p}$. Ioo de ses protéines. Il est cependant permis de supposer que les $20 \mathrm{p}$. Ioo qui échappent à notre analyse se comportent d'une manière analogue vis-à-vis des phénomènes étudiés. De toute façon, la perte d'information relativement minime résultant de 1'usage de cette méthode, est largement compensée par les avantages présentés par ailleurs. En effet, le filtrat parfaitement limpide qu'elle permet d'obtenir, même en présence de farine se prête à un dosage très simple et précis des produits solubilisés. Il eut certes été possible de précipiter les protéines par un réactif donnant de meilleurs résultats (acide trichloracétique, acide perchlorique), mais, avec, en présence de farine, un filtrat trouble, excluant des mesures spectrophotométriques directes et impliquant donc de nombreux dosages d'azote de précision moindre.

En ce qui concerne la protéolyse d'un mélange de caséine ou de gluten, et de blé ou d'extraits de diverses de ses fractions, nous avons constaté qu'elle pouvait être différente de celle de la caséine ou du gluten seuls. Le fait qu'elle puisse être supérieure et qui s'observe avec le gluten, lorsque l'apport protéique de l'effecteur est relativement important, mais peu ou pas avec la caséine, s'explique par l'augmentation de la concentration de substrat. En effet, dans les conditions de concentrations de substrat (Io $\mathrm{mg} / \mathrm{ml}$ ) et d'enzyme $(4 \mu \mathrm{g} / \mathrm{ml}$ ) généralement utilisées, l'augmentation de la concentration de substrat entraîne une augmentation de la pro- 
téolyse pour le gluten, et, du fait d'une inhibition par excès de substrat, une diminution pour la caséine dont la protéolyse est à son maximum. I,e fait que la protéolyse puisse être inférieure et qui se manifeste aussi bien avec la caséine qu'avec le gluten, s'explique par une inhibition de leur protéolyse par certains constituants du blé. Par conséquent, l'effet observé sur la protéolyse serait la résultante de deux actions tendant, l'une à l'augmenter et l'autre à la diminuer dans le cas du gluten, et toutes deux à la diminuer dans le cas de la caséine.

Cette inhibition se manifeste avec le blé et ses extraits aqueux effectués en milieu acide. Elle se manifeste aussi avec les extraits de gros son et de son fin, mais non plus avec celui de farine à 65 p. Ioo. L'extrait de gros son produit une inhibition presque égale à celle du blé, tandis que celle produite par l'extrait de son fin est beaucoup plus faible.

Cette inhibition qui augmente avec la concentration d'extrait est indépendante de la concentration d'enzyme et tend à s'annuler lorsqu'augmente la concentration de substrat. L'acide phytique pur produit aussi une inhibition présentant les mêmes caractéristiques. Nous pouvons donc penser que l'inhibition produite par le blé est due à l'acide phytique présent principalement dans ses issues.

L'inhibition produite par l'acide phytique s'explique par sa combinaison avec les protéines sous forme de phytates de protéines, insolubles à un $\mathrm{pH}$ inférieur au point isoélectrique de ces protéines, et plus résistants que celles-ci à l'attaque de la pepsine. Cette combinaison de 1'acide phytique avec les protéines est un phénomène connu, ayant fait l'objet de nombreux travaux et ayant des applications pratiques (AGNERAY, I965 ; BARRE, I95I, I953 ; BARRE et CoURTOIS, I953 ; BARRE et al., I954 ; Courtors et BARRE, I949, I953 ; Courtors et DELRIEU, I958 ; CourTOIS et PFRLES, I959-I960 ; Courtois et al., I959; DeIrIEU, I958 ; Heliot et MACHEBCEUF, I947; IABAT, I96I). Chacune des six fonctions acides les plus fortes de l'acide phytique se combine à un groupe basique des protéines, en particulier ceux de l'argınine, de la lysine et de l'histidine, ce qui entraîne une agglomération de six groupes basiques autour du noyau cyclohexanique de l'acide phytique, d'où des repliements ou des écartements des séquences polypeptidiques des protéines conduisant à l'insolubilisation des composés formés. Nous avons effectivement constaté que dans le cas de la caséine soluble à $\mathrm{pH}$ 2,0, l'adjonction d'extrait de blé ou de ses issues, d'acide phytique, entraîne une précipitation. Dans le cas du gluten déjà insoluble, l'effet observé est moins net mais se manifeste néanmoins par la formation d'agglomérats. La diminution de l'inhibition entraînée par l'augmentation de la concentration de substrat s'explique par la saturation progressive de l'acide phytique par le substrat protéique.

La pepsine étant de nature protéique, on pourrait aussi penser à une action de l'acide phytique sur l'enzyme. Or, nous avons vu que l'inhibition est indépendante de la concentration d'enzyme. De plus, nous avons constaté que l'adjonction d'acide phytique à une solution de pepsine, n'entraîne à $\mathrm{pH} 2,0$, aucune précipitation. Ceci s'explique par le fait que le point isoélectrique de la pepsine est de l'ordre de $\mathrm{I}$ (Bovey et YANARI, I960). A pH 2,0, elle ne peut donc donner lieu à la formation de phytate de pepsine insoluble. Une action, n'allant pas jusqu'à la précipitation. de l'acide phytique sur la pepsine est cependant possible.

En ce qui concerne l'extraction du facteur inhibant la protéolyse pepsique, c'est-à-dire l'acide phytique, si les courbes que nous avons obtenues en fonction du 
$\mathrm{pH}$ d'extraction montrent une inhibition maximum aux $\mathrm{pH}$ les plus faibles tant pour la caséine que pour le gluten, la concordance ne se maintient plus lorsque le pH augmente. L'effet observé peut cependant s'expliquer, d'une part, par ce que nous savons par ailleurs des teneurs moyennes du blé en acide phytique et de son extraction en fonction du $\mathrm{pH}$ (COURTOIS et PEREZ, I948; GUILLEMET, I946 ; KENTJONES et Amos, I950), et d'autre part, par ce que nous avons observé quant à l'inhibition par l'acide phytique pur de la protéolyse pepsique de la caséine et du gluten (fig. 6). D'après MNICH (I926), l'extraction de 1'acide phytique du blé, peu importante en milieu aqueux voisin de la neutralité, augmente avec l'acidification, avec cependant un minimum d'extraction vers $\mathrm{pH} 3,0$ où se forme le maximum de combinaisons insolubles avec les protéines. Nos courbes de la figure 3 mettent effectivement en évidence ce minimum d'extraction à $\mathrm{pH} 3,0$, par un minimum d'inhibition pour le gluten mais non pour la caséine. Cette divergence feut s'expliquer par les différences entre les courbes d'inhibition par 1'acide phytique pur des protéolyses de la caséine et du gluten (fig. 6) : il existe une zone critique de concentration d'acide phytique se situant vers $5 \mathrm{mg} / \mathrm{ml}$ de phytate de sodium, telle que, bien que l'inhibition ait atteint son maximum dans les deux cas, une diminution de la concentration d'acide phytique entraîne une diminution de l'inhibition pour le gluten mais non pour la caséine. Or, un calcul approximatif de la concentration possible d'acide phytique dans nos expériences de son extraction en fonction du $\mathrm{pH}$, la situe effectivement dans cette zone critique.

Les résultats obtenus avec le blé Étoile de Choisy sont confirmés par ceux obtenus avec les blés Rex et $28 p C$ rouge. Comme par ailleurs nous savons que les teneurs en acide phytique du blé peuvent varier dans un rapport de I à 2 (CourToIs et Perez, I948 ; GuIlleme', I946 ; Kent-Jones, I950), on peut penser que 1'inhibition de la protéolyse par les blés que nous avons étudiés, se retrouverait avec peu de modifications pour l'ensemble des blés. On peut aussi penser que tout aliment renfermant de l'acide phytique serait susceptible de produire une telle inhibition. L,e problème se pose donc des conséquences nutritionnelles possibles d'une telle inhibition. Jusqu'à présent, l'acide phytique était essentiellement considéré comme ayant une influence défavorable sur l'absorption du calcium, du magnésium et du fer (GUILLEMET, I946; JACQUOT, I957; KENT-JoNES et Amos, I950), du fait de la formation avec ces divers éléments, de composés insolubles en milieu neutre ou alcalin. Il ne semble pas que 1'on ait envisagé son intervention dans la protéolyse pepsique stomacale, bien que la formation avec les protéines de composés insolubles en milieu acide à laquelle il donne lieu, soit aussi un phénomène très connu. Cependant, l'intervention de l'acide phytique avait été envisagée (JoRDAN et al., rgo6) pour expliquer l'augmentation de l'azote fécal produite par le son de blé. Mais cette hypothèse n'ayant pas été confirmée par ailleurs (CoWGILL et ANDERSON, I932; MENDEL, et UNDERHILL, I906; WILLIAMS, I927), cette action est le plus habituellement attribuée à 1'indigestible glucidique (JACQUOT, I957) et notamment à la lignine (CHARLET-L,ERY et al., 1953). Cependant, selon FourNIER (I948, I949), l'augmentation de l'azote fécal serait surtout d'origine bactérienne, l'apport par le son de vitamines et oligo-éléments favorisant le développement de bactéries dont certaines aux dépens de l'indigestible glucidique. Les problèmes nutritionnels posés par l'acide phytıque tant du blé que des autres aliments en renfermant, sont d'ailleurs en relation étroite avec la teneur en phytases associées (Courtors et 
Perez, I948; Hiller, I968; Kent-Jones et AMOS, I950) et en les possibilités d'action préalable de ces phytases (Cour'Tors et VALEn'rino, I947), la présence éventuelle de phytases endogènes ou d'origine microbienne dans le tube digestif, ne semblant pas devoir jouer dans le cas présent, du fait de leur localisation intestinale. Quoi qu'il en soit, nous nous proposons d'étudier ultérieurement in vivo, l'effet d'une surcharge du régime en acide phytique.

Reçu pour publication en septembre 1975.

\section{REMERCIEMENTS}

Le gluten de blé Étoile de Choisy nous a été très aimablement fourni par M. P. FErLLET, directeur du Laboratoire de technologie des blés durs de Montpellier, et les différents blés et leurs différentes fractions de meunerie par M. P. GRandvolner, directeur scientifique aux Grands Moulins de Pantin.

Nous les en remercions très vivement ainsi que M. P. Fournier, directeur du Laboratoire du métabolisme minéral des mammifères de l'École Pratique des Hautes Études, pour l'intérêt qu'il a porté à ce travail.

\section{SUMMARY}

\section{WHEAT AS AN IN VITRO INHIBITOR OF PEPTIC PROTEOLYSIS. ROLE OF PHYTIC ACID IN BY-PRODUCTS}

The peptic proteolysis of casein or gluten is examined in vitro. It is studied pure or supple. mented with Étoile de Choisy wheat, aqueous extracts of that wheat or of its various milling fractions.

Proteolysis is done at $\mathrm{pH} 2.0$ and products solubilized in $0.1 \mathrm{M}$ acetic acid and sodium acetate ethanolic solution are determined spectrophotometrically at wave length $280 \mathrm{~nm}$. Proteolysis of both casein and gluten is inhibited by wheat, its extracts in acid medium and coarse and fine bran acid extracts. This did not occur using 65 p. roo flour acid extracts.

Inhibition depends on concentrations of both substrate and inhibiter (increase with inhibitor concentration, decrease as substrate concentration increases); it is independent of pepsin content. The same phenomena are obtained with $R e x$ and $28 p C$ rouge wheats. This inhibition seems to be due to the phytic acid mainly present in by-products; when added in a pure form, it produces an inhibition with similar characteristics.

Peptic proteolysis inhibition by phytic acid is explained by the formation of insoluble protein phytates in acid medium. These are more resistent than proteins to pepsin action.

\section{RÉFÉRENCES BIBLIOGRAPHIQUES}

AgNERAY J., 1965. Fractionnement des protéines du sérum sanguin à l'aide de l'acide inositohexaphosphorique. Thèse Sciences, Lille.

Barre R., I95I. Préparation et purification des protéines des amandes. Bull. Soc. Chim. Biol., 33, I473-I 482 .

BARRE R., I953. Recherches sur les protéines des graines d'amandiers et leurs combinaisons avec divers dérivés phosphorés. Thèse Sciences, Paris.

Barre R., Covrtois J. E., I953. Recherches sur les combinaisons des protéines avec les composés phosphorés. II. Étude des combinaisons de l'acide $\beta$-glycérophosphorique et de l'acide phytique. Bull. Soc. Chim, Biol, , 35, $921-932$. 
Barre R., Courtois J. E., Wormser G., I954. Étude de la structure de l'acide phytique au moyen de ses courbes de titration et de la conductivité de ses solutions. Bull. Soc. Chim. Biol., 36, 455-474.

Barre R., Courtois J. E., Delrieu P., Perles R., r954. Emploi de l'acide phytique pour la détermination des groupements basiques des protéines dans les sérums normaux et pathologiques. $A n n$. Biol. Clin., 12, 501-509.

Bourdet A., r956. Les protides des céréales. Ann. Technol., 5, 181-318.

Bovey F. A., Yanari S. S., rg6o. Pepsin in Boyer P. D., Lardy H., Myrbäck K., The Enzymes, 4 A, 63-92, ze éd., Acad. Press, New York.

Camus M. C., Sattier C., 1972. Protéolyse pepsique d'aliments d'origine animale et végétale. Ann. Biol. anim. Bioch. Biophys., 12, $28 \mathrm{I}-288$.

Charley-Lery G., François A., Leroy A. M., I952. Analyse des aliments destinés aux animaux et l'interprétation des résultats qu'elle fournit.'Ann. Zootech., 1 (3) 45-6I.

Courtors J. E., BARRe R., I949. Influence de la réaction du milieu sur la précipitation de quelques esters phosphoriques avec une protéine des amandes. Bull. Soc. Chim. Biol., 31, 740-749.

Courtois J., Barre R., r953. Recherches sur les combinaisons des protéines avec les composés phosphorés. I. Étude de l'amando-globuline la légumine et l'insuline. Bull. Soc. Chim. Biol., 35, 9r 3-92I.

Courtois J. E., Delrieu P., r958. Emploi de l'acide phytique pour la détermination des groupes basiques des protéines urinaires. Ann. Biol. Clin., 16, 29-38.

Courtois J., Perez. C., r948. Teneur en inositophosphates, et activité phytasique de diverses graines. In Journées Scientifiques du C.N.E.R.N.A., Le pain, I43-I52, C.N.R.S., Paris.

Courtois J. E., Perles R., r959-1960. Précis de Chimie Biologique, 2 vol., Masson, Paris.

Courtois J. E., Valentino A., 1947. Recherches sur la phytase. IV. L'ingestion de phytase peut-elle favoriser l'absorption des inositophosphates? Bull. Soc. Chim. Biol., 29, 615-620.

Courtors J. E., Malangeau P., Chabre J. L., r959. Recherches sur les combinaisons des protéines avec les composés phosphorés. V. Influence des glycoprotéides sur la précipitation phytique des holoprotéines. Ann. Pharm. Franç., 27, 561-57o.

Cowgill G. R., ANDERSon W. E., 1932. Laxative effects of wheat bran and " was hed bran 》 in healthy men. J. Amer. Med. Assoc., 98, 1866-1875.

DeLriev P., 1958. Emploi de l'acide phytique pour l'étude des groupements basigues des protéines du sérum sanguin et de l'urine. Thèse Pharmacie, Paris.

Fournier P., 1948. Les constituants des membranes végétales dans leurs rapports avec les phênomènes digestifs. Contribution à l'étude de leur influence sur la nutrition chez l'Homme. Thèse Sciences, Paris.

Fournier P., 1949. Relations entre l'ingestion de membranes végétales et l'excrétion d'azote fécal. Bull. Soc. Chim. Biol., 31, 407-4II.

Fournier P., I949. Origines de la surproduction d'azote fécal provoquée par l'ingestion de son de blé. Bull. Soc. Chim. Biol., 81, 4I I-4I7.

Gulllemet R., I946. L'acide phytique. Biol. Méd., 35, I-21.

Hellot R., Machebeuf M., r947. Les protéides de la graine d'arachide (Avachis hypogea). Identification des impuretés phosphorées. Bull. Soc. Chim. Biol, 29, 811-822.

Hiller A., 1968. Répercussion de la richesse en phytase sur la teneur du pain en phytine. Industr. Alim. A gr., 85, 27-3I.

Jacquot R., I957. Les facteurs d'efficacité alimentaire. In Autret M., Ganzin M., Nutrition et Alimentation tropicales, 1, 323-433, F.A.O., O.M.S., Rome.

Jordan W. H., Hart E. B., Patten A. J., 1906. A study of the metabolism and physiological effects of certain phosphorus compounds with milk cows. Amer. J. Physiol., 16, 268-313.

Kent-Jones D. W., Amos A. J., r950. Modern Cereal Chemistry, 4 éd, The Nothern Publishing Co., Liverpool.

Labat J., 1961. Étude de la précipitation des protéines des liquides biologiques par l'acide phytique. Thèse Pharmacie, Paris.

LAPORTE J. C., I964. Mise en évidence et étude de facteurs antiprotêolytiques dans les cêréales. Thèse $3^{\mathrm{e}}$ cycle, Paris.

MENDEL L. B., UNDERHILL F. P., I906. Experiments on the physiological action and metabolism of an hydro-oxymethylene-diphosphoric acid (phytin acid). Amer. J. Physiol., 17, 75-88.

Mnich E., 1926. Bull. Int. Ac. Pol. Sc. et Let., B, r23 (d'après Guillemet, Hellot et Machebauf; Courtois et Barre déjà cités).

Williams G. A., I927. A study of the laxative action of wheat bran. Amer. $J$. Physiol., 83, I-I4. 